\title{
Low-mass X-ray Binaries with Strange Quark Stars
}

\author{
Chunhua Zhu ${ }^{1,2,3}$, Guoliang Lü $^{1,2}$, Zhaojun Wang ${ }^{1,2}$, Jinzhong Liu $^{3}$ \\ ${ }^{\dagger}$ chunhuazhu@sina.cn
}

\begin{abstract}
Strange quark stars (SSs) may originate from accreting neutron stars (NSs) in lowmass X-ray binaries (LMXBs). Assuming that conversion of NS matter to SSs occurs when the core density of accreting NS reaches to the density of quark deconfinement, $\sim 5 \rho_{0}$, where $\rho_{0} \sim 2.7 \times 10^{14} \mathrm{~g} \mathrm{~cm}^{-3}$ is nuclear saturation density, we investigate LMXBs with SSs (qLMXBs). In our simulations, about 1\%o- $10 \%$ of LMXBs can produce SSs, which greatly depends on the masses of nascent NSs and the fraction of transferred matter accreted by the NSs. If the conversion does not affect binaries systems, LMXBs evolve into qLMXBs. We find that some observational properties (spin periods, X-ray luminosities and orbital periods) of qLMXBs are similar with those of LMXBs, and it is difficult to differ them. If the conversion disturbs the binaries systems, LMXBs can produce isolated SSs. These isolated SSs could be submillisecond pulsars, and their birthrate in the Galaxy is $\sim 5-70$ per Myr.

Subject headings: $\mathrm{b}$

inaries: close -stars: neutron-dense matter
\end{abstract}

\section{Introduction}

There are at least three different kinds of compact stars in the Universe: white dwarfs (WDs), neutron stars (NSs), and black holes. Witten (1984) suggested a possible existence of compact objects consisting of strange quark matter. Due to strange quark matter being absolutely stable, Haensel et al. (1986) and Alcock et al. (1986) pointed out that NSs almost would be made of strange matter and not neutrons. However, Alpar (1987) considered that glitching radio-pulsars are NSs and not strange quark stars (SSs). Madsen (1988) suggested that SSs can not be formed directly in supernovat 1 , or less they would eventually contaminate the entire Galaxy. Kluzniak (1994)

\footnotetext{
${ }^{1}$ School of Physical Science and Technology, Xinjiang University, Urumqi, 830046, China.

${ }^{2}$ Xinjiang University-National Astronomical Observatories Joint Center for Astrophysics, Urumqi, 830046, China

${ }^{3}$ National Astronomical Observatories / Xinjiang Observatory, the Chinese Academy of Sciences, Urumqi, 830011, China

${ }^{1}$ Dai et al. (1995) and Xu et al. (2001) suggested that SSs can be formed directly during or shortly after some supernovae explosion when the central density of the proto-NSs is high enough.
} 
suggested that SSs could exist as millisecond pulsars. Due to the fast rotation and thermonuclear bursts, Li et al. (1999) suggested that the SAX J1808.4-3658 is a good SS candidate. These SSs can be formed in low-mass X-ray binaries (LMXBs) via an accretion-triggered phase transition of NS matter to SS matter (Cheng \& Dai 1996).

The phase transition requires the formation of a strange matter seed in the NS. The strange matter is produced through the neutron matter at a critical density. Serot \& Uechi (1987) pointed out that the central density of an 1.4 $M_{\odot}$ NS with a rather stiff equation of state is sufficiently lower than the critical density. Based on the modern equations of state in Wiringa et al. (1988), Cheng \& Dai (1996) estimated that the NSs with $1.4 M_{\odot}$ must accrete matter of $\sim 0.5 M_{\odot}$ in order that their central densities reach the deconfinement density. Once the above condition is satisfied, the phase transition occurs.

Olinto (1987) proposed that the process of the strange matter swallowing the neutron matter is a slow mode. However, Horvath \& Benvenuto (1988) showed that it is hydrodynamically unstable. Cheng \& Dai (1996) proposed that the conversion of neutron matter should proceed in a detonation mode and could be accompanied by a gamma-ray burst. Ouved et al. (2002) suggested that there is a quark-nova when the core of a NS (having experienced a transition to an up and down quark phase) shrinks into the equilibrated quark object after reaching strange quark matter saturation density (where a composition of up, down and strange quarks is the favored state of matter). In their model, the energy released as radiation in a quark-nova is up to $10^{53} \mathrm{ergs}$. Ouved et al. (2011) proposed that the quark novae in LMXBs may be the engines of short gamma-ray bursts.

Based on the above descriptions, it is possible that SSs originate from the hydrodynamically unstable conversion or the slow conversion in LMXBs. Using standard equation of states of neutron-rich matter, Staff et al. (2006) considered that the density of quark deconfinement is $\sim 5 \rho_{0}$, where $\rho_{0} \sim 2.7 \times 10^{14} \mathrm{~g} \mathrm{~cm}^{-3}$ is nuclear saturation density. According to the equation of states in Akmal et al. (1998), the gravitational mass of a NS is $\sim 1.8 M_{\odot}$ in order to reach $5 \rho_{0}$. Therefore, it is very important for our understanding of SSs' formation to study the mass evolution of NSs in LMXBs.

In this work, by simulating the interaction of a magnetized NS with its environment and utilizing a population synthesis code, we focus on the mass change of NSs in LMXBs and the possibility from NSs converting SSs in LMXBs, and investigate the properties of LMXBs with SSs (qLMXBs). In Section 2, we present our assumptions and describe some details of the modelling algorithm. In Section 3, we discuss the main results and the effects of different parameters. In Section 4 , the main conclusions are given.

\section{Model}

For the simulation of binary evolution, we use rapid binary star evolution code BSE (Hurley et al. 2002) which was updated by Kiel \& Hurley (2006). In interacting binaries, NSs can be formed via 
three channels(e.g., Ivanova et al. 2008; Kiel et al. 2008): (i) Core-collapse supernovae (CCSN) for a star; (ii)Evolution induced collapse (EIC) of a helium star with a mass between 1.4 and $2.5 M_{\odot}$ in which the collapse is triggered by electron capture on ${ }^{20} \mathrm{Ne}$ and ${ }^{24} \mathrm{Mg}$ (Mivaji et al. 1980); (iii) Accretion-induced collapses (AIC) for an accreting ONeMg WD whose mass reaches the Chandrasekhar limit. Response of accreting $\mathrm{ONeMg}$ WD is treated in the same way as the evolution of CO WD (see details in Lü et al. 2009).

\subsection{Mass of Nascent NS}

Possibly the mass is one of the most important properties of NSs. However, the mass distribution of nascent NSs is not yet well known. In BSE code, the gravitational mass of a nascent NS via CCSN depends on the mass of the CO-core at the time of supernova (Hurlev et al. 2000). Figure 1 shows the masses of nascent NSs forming from different initial masses. Some authors assumed that the initial masses of NSs $\left(M_{\mathrm{NS}}^{\mathrm{i}}\right)$ are $1.4 M_{\odot}$ in their works (Ergma et al. 1998; Podsiadlowski et al. 2002; Nelson \& Rappaport 2003, e.g.,). Lattimer \& Prakash (2007) showed that the masses of some NSs are lower than 1.4 $M_{\odot}$. Recently, van der Meer et al. (2007) found that the masses of NSs in SMC X-1 and Cen X-3 are $1.06_{-0.10}^{+0.11} M_{\odot}$ and $1.34_{-0.14}^{+0.16} M_{\odot}$, respectively. However, It is well known that most of the accurately measured masses of NSs are near $1.4 M_{\odot}$.

In our work, we use the initial masses of NSs via CCSN in Hurley et al. (2000) and $M_{\mathrm{NS}}^{\mathrm{i}}=$ $1.4 M_{\odot}$ in different simulations, respectively. For NSs via AIC, following Hurley et al. (2000), we take $M_{\mathrm{NS}}^{\mathrm{i}}=1.3 M_{\odot}$. Similarly, for NSs via EIC, we also take $M_{\mathrm{NS}}^{\mathrm{i}}=1.3 M_{\odot}$.

In addition, nascent NS receives additional velocity ("kick") due to some still unclear process that disrupts spherical symmetry during the collapse or later Dichotomous nature of kicks which was suggested quite early by Katz (1975). Observationally, the kick is not well constrained due to numerous selection effects. Currently, high kicks $\left(\sim 100 \mathrm{~km} \mathrm{~s}^{-1}\right)$ are associated with NS originating from CCSN, while low kicks $\left(\sim 10 \mathrm{~km} \mathrm{~s}^{-1}\right)$ with NS born in EIC and AIC (Pfahl et al. 2002). We apply to core-collapse NS Maxwellian distribution of kick velocity $v_{\mathrm{k}}$

$$
P\left(v_{\mathrm{k}}\right)=\sqrt{\frac{2}{\pi}} \frac{v_{\mathrm{k}}^{2}}{\sigma_{\mathrm{k}}^{3}} e^{-v_{\mathrm{k}}^{2} / 2 \sigma_{\mathrm{k}}^{2}} .
$$

Variation of $\sigma_{\mathrm{k}}$ between 50 and $200 \mathrm{~km} \mathrm{~s}^{-1}$, introduces an uncertainty $\leqslant 3$ in the birthrate of lowand intermediate-mass X-ray binaries (Pfahl et al. 2003). Zhu et al. (2012) discussed the effects of parameter $\sigma_{\mathrm{k}}$ on LMXBs' populations. Since in this paper we focus on the physical parameters that mostly affect the masses of NSs, we do not discuss the effects of $\sigma_{\mathrm{k}}$ on SSs' population. Following Lü et al. (2012), we take $\sigma_{\mathrm{k}}=190 \mathrm{~km} \mathrm{~s}^{-1}$ in CCSN, and $\sigma_{\mathrm{k}}=20 \mathrm{~km} \mathrm{~s}^{-1}$ in EIC and AIC. 


\subsection{Mass of Accreting NS}

In LMXBs, NSs accrete the matter from their companions via Roche lobe flows or stellar winds. The interaction of a rotating magnetized NS (single or in a binary system) with surrounding matter has been studies by many authors (Pringle \& Rees 1972; Illarionov \& Sunyaev 1975; Ghosh \& Lamb 1978; Lovelace et al. 1995, 1999, e. g.,).

Using a convenient way of describing NS evolution elaborated by Lipunov et al. (1992) and a recent model for quasi-spherical accretion including subsonic settling proposed by Shakura et al. (2012), Lü et al. (2012) gave detailed simulations for spin period evolution and matter accretion

of NSs in binaries. In this work, we adopt their model. Lü et al. (2012) assumed that all matter transferred is accreted by the NS in an accretor stage. We introduce a parameter $\beta$ which is the fraction of transferred matter accreted by the NS, and the rest of the transferred matter is lost from binary system. The lost matter takes away the specific angular momentum of the prospective donor. The value of $\beta$ has been usually set to 0.5 (Podsiadlowski et al. 1992, 2002; Nelson \& Rappaport 2003). In our work, we set $\beta=0.1,0.5$ and 1.0 in different simulations.

\subsection{LMXBs with Nascent SSs}

As noted in 91 , when the gravitational mass of a NS reaches to $\sim 1.8 M_{\odot}$, the NS can turn into a SS via the hydrodynamically unstable conversion or the slow conversion. In the former mode, the binary system may be disrupted (Ouved \& Staff 2011), and it becomes two isolated stars. One of them is an isolated SS. However, it is difficult to know the effects of the hydrodynamically unstable conversion on binary systems. Therefore, in our work, we consider two extreme cases: (i) In order to simulate all potential properties of qLMXBs, we assumed all LMXBs are not affected and survive after the conversion, that is, LMXBs become qLMXBs when the masses of accreting NSs are larger than $1.8 M_{\odot}$; (ii) We assumed that all LMXBs are disrupted after the conversion, that is, there is no qLMXBs. However, we can discuss the origin of isolated submillisecond pulsars.

In our simulations, if a nascent NS has larger mass than $1.8 M_{\odot}$ it is a SS. Therefore, in the paper, qLMXBs include some LMXBs in which the nascent NSs have larger masses than $1.8 M_{\odot}$. Lai \& Xu (2009) suggested that SSs could have high maximum masses (See Figure 2 in Lai \& Xu (2009)) if they are composed of the Lennard-Jones matter. In our work, we assume that the maximum mass of SS is $3.0 M_{\odot}$.

\section{Results}

We use Monte Carlo method to simulate the initial binaries. For initial mass function, massratios, and separations of components in binary systems, we adopt the distributions used by us in Lü et al. (2006, 2008). We assume that all binaries have initially circular orbits. After a supernova, 
new parameters of the orbit are derived using standard formulae(e. g., Hurley et al. 2002). It is well known that theoretical models of the population of LMXBs depend on badly known input parameters, such as kick velocity and common envelope treatment (e. g., Pfahl et al. 2003; Zhu et al. 2012). However, in this pioneering study of qLMXBs we focus on the effects which are important for the masses of NSs: the efficiency accreted of transferred matter, $\beta$ ( $\beta=0.1,0.5$ and 1.0 ), and the mass of nascent NS via CCSN (See Figure 1 ). We use $1 \times 10^{8}$ binary systems in our Monte-Carlo simulations.

\subsection{Mass Increases of Accreting NSs}

Figure 2 gives the mass increases of accreting NSs in LMXBs. According the assumptions of SSs formatting from NSs with masses higher than $1.8 M_{\odot}, 1 \%$ o $(\beta=0.1)-10 \%(\beta=1.0)$ of LMXBs are qLMXBs in our simulations. This proportion greatly depends on input parameters $\beta$ and the initial masses of nascent NSs. In the cases of $\beta=1.0$ and $\beta=0.5$, most of SSs in qLMXBs come from low-mass NSs with $1.4 M_{\odot}$, that is, they have accreted $\sim 0.4 M_{\odot}$ matter. In the case of $\beta=0.1$, most of SSs in qLMXBs originate from CCSN.

Mass increases of accreting NSs in LMXBs depend on not only input parameter $\beta$ in this work, but also orbital periods and NSs' companions. Zhu et al. (2012) showed that most of NSs in LMXBs with WD donors have low mass-accretion rates $\left(\sim 10^{-12} \dot{M}_{\odot} \mathrm{yr}^{-1}\right)$ and most of LMXBs with WD donors are transient. Therefore, the masses of accreting NSs in LMXBs with WD donors hardly reach to $1.8 M_{\odot}$. Less than $10 \%$ (in case of $\beta=0.1$ ) $1 \%$ (in case of $\beta=0.5, M_{\mathrm{NS}}^{\mathrm{i}}=1.4 M_{\odot}$ ) of qLMXBs have WD donors in our simulations. Most of qLMXBs have main sequence donors. From now on, we just discuss LMXBs and qLMXBs with MS donors.

\subsection{Properties of qLMXBs}

According to our assumption that the conversion of NS matter to SS matter does not affect binary systems, we can simulate some observational properties of LMXBs and qLMXBs, and wish to find some differences between them. Then, taking the case of $\beta=0.5$ as an example, we discuss some properties of qLMXBs.

X-ray luminosities (mass-accretion rates), spin periods and orbital periods are important parameters of LMXBs. Figure 3 shows the accretion rates by NSs or SSs in LMXBs and qLMXBs, and the X-ray luminosities which are approximated as

$$
L_{\mathrm{x}}=\eta \dot{M}_{\mathrm{NS}} \mathrm{c}^{2} \simeq 5.7 \times 10^{35} \mathrm{erg} \mathrm{s}^{-1}\left(\frac{\eta}{0.1}\right) \times\left(\frac{\dot{M}_{\mathrm{NS}}}{10^{-10} M_{\odot} \mathrm{yr}^{-1}}\right),
$$

where $\eta \simeq 0.1$ is the efficiency of converting accreted mass into X-ray photons. We can find that there is not significant difference between accretion rates by NSs or SSs in LMXBs and qLMXBs. 


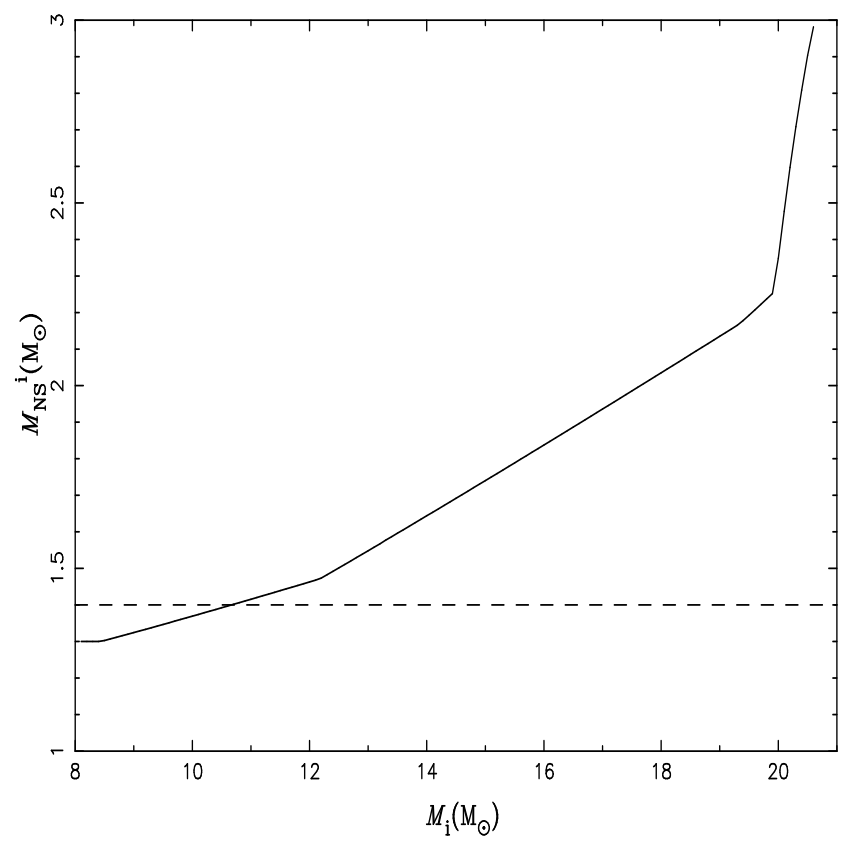

Fig. 1.- Masses of nascent NSs via CCSN vs. stellar initial masses. The solid line comes from Hurley et al. (2000), and the dashed line means that masses of nascent NSs are equal to $1.4 M_{\odot}$.

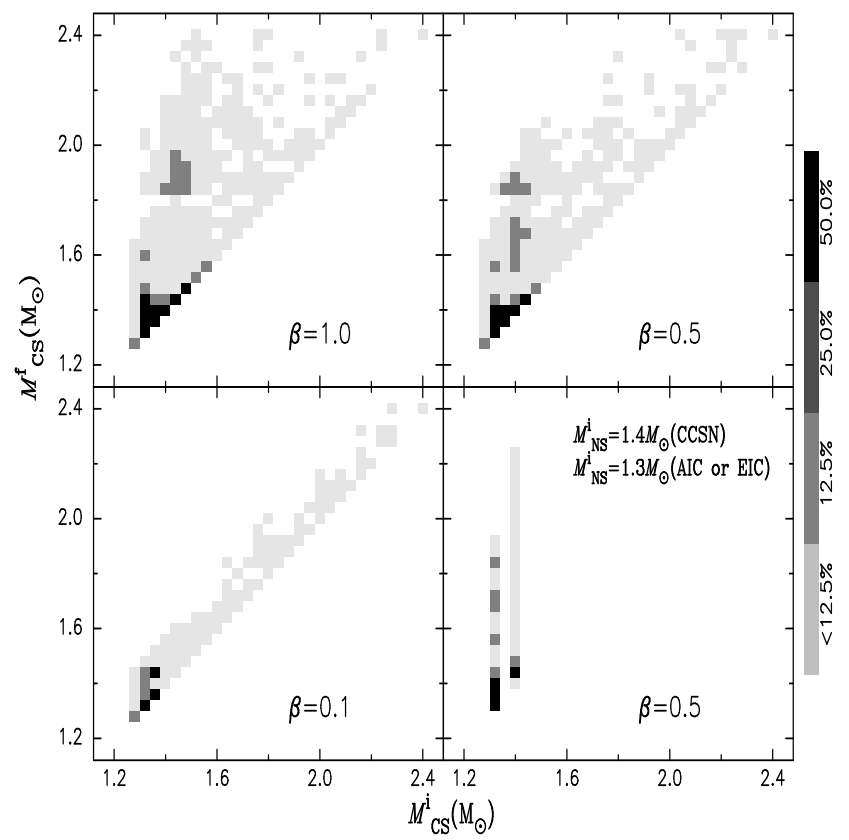

Fig. 2.- Distributions of the final masses of compact stars (NSs or SSs) vs. their initial masses in LMXBs for different input parameters. Gradations of gray-scale correspond to the number density of systems $>1 / 2,1 / 2-1 / 4,1 / 4-1 / 8,1 / 8-0$ of the maximum of $\frac{\partial^{2} N}{\partial M_{\mathrm{CS}}^{\mathrm{i}} \partial M_{\mathrm{CS}}^{\mathrm{f}}}$ and blank regions do not contain any stars. 
The spin periods of the rotating magnetized NSs mainly depend on their mass-accretion rates (Lü et al. 2012). If accreting SSs are similar to NSs, we can simulate the spin periods of accreting SSs. Figure 4 gives the distribution of the spin periods of NSs in LMXBs or SSs in qLMXBs. In general, spin periods of SSs in qLMXBs are shorter than those of NSs in LMXBs.

Figure 5 shows the the distributions of the orbital periods $P_{\text {orb }}$ of qLMXBs and LMXBs vs. masses of their secondary stars. Similarly, there is not significant difference between qLMXBs and LMXBs.

As the above descriptions show, it is very difficult in our model to differ from LMXBs and qLMXBs. The most effective way is to measure the masses of compact objects in LMXBs if the assumption that NSs with masses larger than $1.8 M_{\odot}$ are SSs is right.

Jonker et al. (2005) suggested that compact object in 2S 0921-630 (It is a LMXB) has a mass between $\sim 1.9-2.9 M_{\odot}$. According to our assumption, the compact object is a SS. However, Steeghs \& Jonker (2007) considered that Jonker et al. (2005) overestimated the rotational broadening and the mass of compact object in 2S 0921-630 is $\sim 1.44 M_{\odot}$. The orbital period of 2S 0921-630 is $9.006 \pm 0.007$ days, and its X-ray luminosity is $\sim 10^{36} \mathrm{erg} \mathrm{s}^{-1}$ (Kallman et al. 2003). Results of simulating LMXBs and qLMXBs in our work both cover these observations. Therefore, we can not conclude whether compact object in 2S 0921-630 is NS or SS.

Demorest et al. (2010) gave that PSR 1614-2230 has a mass of $1.97 M_{\odot}$. And, it is a millisecond radio pulsar (Pulsar spin period is $3.15 \mathrm{~ms}$ ) in an 8.7 day orbit, and its companion has a mass of $0.5 M_{\odot}$. Although PSR 1614-2230 is not X-ray binary, it may come from X-ray binary. Lin et al. (2011) suggested that PSR 1614-2230 descended from a LMXB very much like Cyg X-2 ( $P_{\text {orb }}=9.8$ days, $M_{\mathrm{NS}}=1.7 M_{\odot}$ and $M_{2}=0.6 M_{\odot}$, see Casares et al. (2010)). AS Figure 5 shows, our results cover the positions of orbital periods and companion masses of Cyg X-2 and radio millisecond pulsar binary PSR 1614-2230. Our work support that PSR 1614-2230 originate from a LMXB. PSR 1614-2230 may be a SS.

\subsection{Submillisecond Pulsars}

Weber (2005) suggested that an isolated submillisecond pulsar spinning at $\sim 0.5 \mathrm{~ms}$ could strongly hint the existence of SSs. If the conversion of NS matter to SS matter is hydrodynamically unstable and LMXBs are disrupted, the nascent SSs are isolated. Assuming that one binary with $M_{1} \geq 0.8 M_{\odot}$ is formed per year in the Galaxy (Yungelson et al. 1993; Han 1998; Hurley et al. 2002), we can estimate that the occurrence rate of hydrodynamically unstable conversion is about 5-70 per Myr.

As Figure 6 shows, the majority of NSs at the beginning of the conversion have spin periods longer than $1 \mathrm{~ms}$. If the angular momentum is conserved and no unknown physical mechanic spins up NSs and nascent SSs during the conversion, the spin periods of nascent SSs depend on 


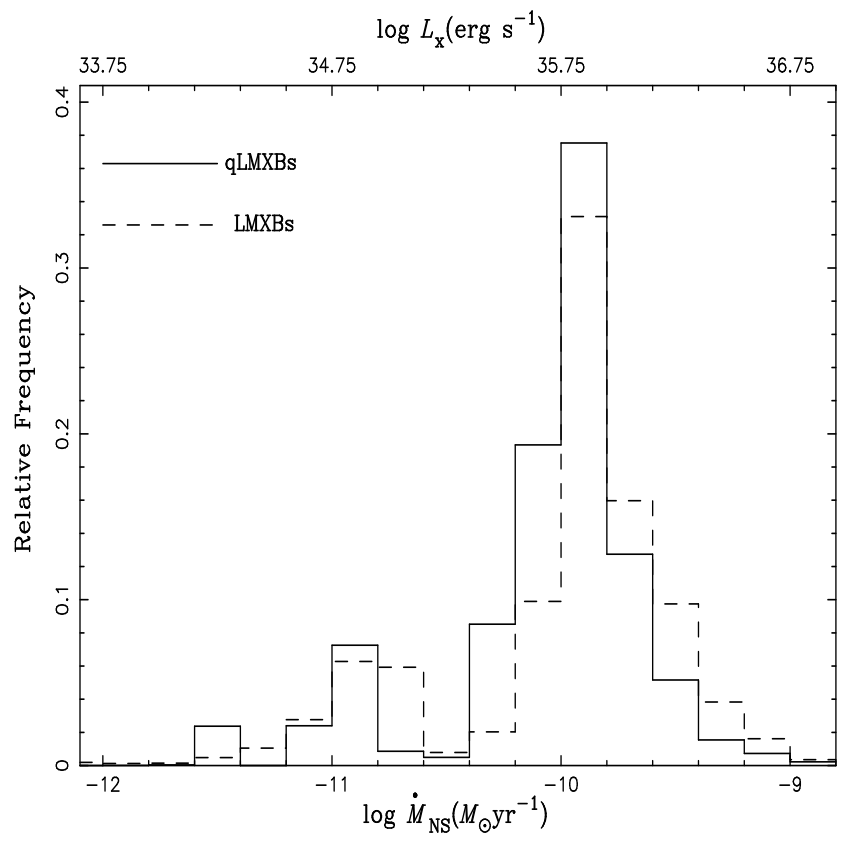

Fig. 3.- Distributions of accretion rates (X-ray luminosities) by NSs or SSs in LMXBs and qLMXBs, respectively.

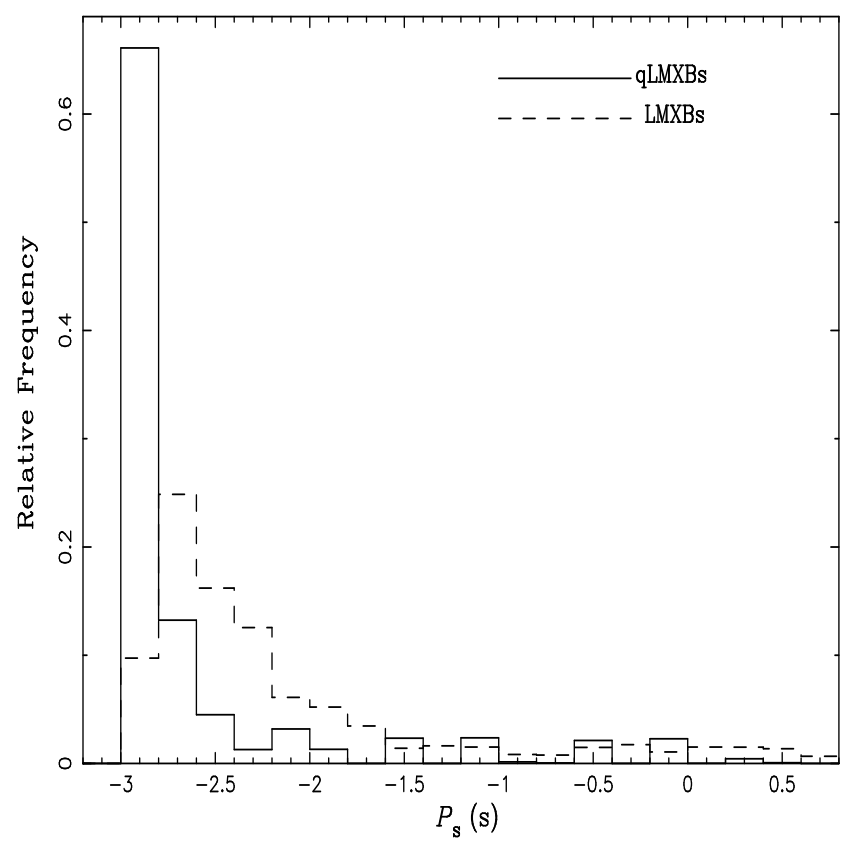

Fig. 4. - Number distributions of the spin periods of NSs or SSs in LMXBs and qLMXBs, respectively. The numbers are normalized to 1 . 
the change of moment of inertia. Ouyed \& Staff (2011) considered that a typical ejected mass of hydrodynamically unstable conversion is $\sim 10^{-3} M_{\odot}$. Therefore, the change of moment of inertia is determined by the difference of radius between NS and SS. Ouved et al. (2002) estimated that NS could shrink by as much as $30 \%$. Then, many nascent SSs have spin periods of $\sim 0.5 \mathrm{~ms}$ in our simulations. However, this result greatly depends on the equation of state of NSs and SSs which are poorly known. If NS only shrinks by as much as $10 \%$ (Private discussion with $\mathrm{Xu}$ ), it is difficult for the nascent SSs to spin up to $0.5 \mathrm{~ms}$.

\section{Conclusions}

Employing the population synthesis approach to the evolution of binaries and using the interacting model of a rotating magnetized NS with surrounding matter, we investigate the mass change of NSs in LMXBs and the possibility from NSs converting SSs in LMXBs. Our results show that about $1 \%$ - $10 \%$ of LMXBs can produce SSs. These SSs may exist in qLMXBs or be isolated, which depends on physical model of the conversion of NS matter to SS mater.

Our toy model can not conclude whether there are SSs in the Galaxy and can not give what properties qLMXBs have. In further work, we need detailed physical model (equation of state about NS and SS and the conversion of NS matter to SS mater) to improve our work.

\section{Acknowledgments}

This work was supported by the National Natural Science Foundation of China under Nos. 11063002 and 11163005, Natural Science Foundation of Xinjiang under Nos.2009211B01 and 2010211B05, Foundation of Huoyingdong under No. 121107, Foundation of Ministry of Education under No. 211198, and Doctor Foundation of Xinjiang University (BS100106).

\section{REFERENCES}

Akmal, A., Pandharipande, V. R., \& Ravenhall, D. G. 1998, Phys. Rev. C, 58, 1804

Alcock, C., Farhi, E., \& Olinto, A. 1986, ApJ, 310, 261

Alpar, M. A. 1987, Phys. Rev. Lett., 58, 2152

Casares, J., González Hernández, J. I., Israelian, G., \& Rebolo, R. 2010, MNRAS, 401, 2517

Cheng, K. S., \& Dai, Z. G. 1996, Physical Review Letters, 77, 1210

Dai, Z., Peng, Q., \& Lu, T. 1995, ApJ, 440, 815 


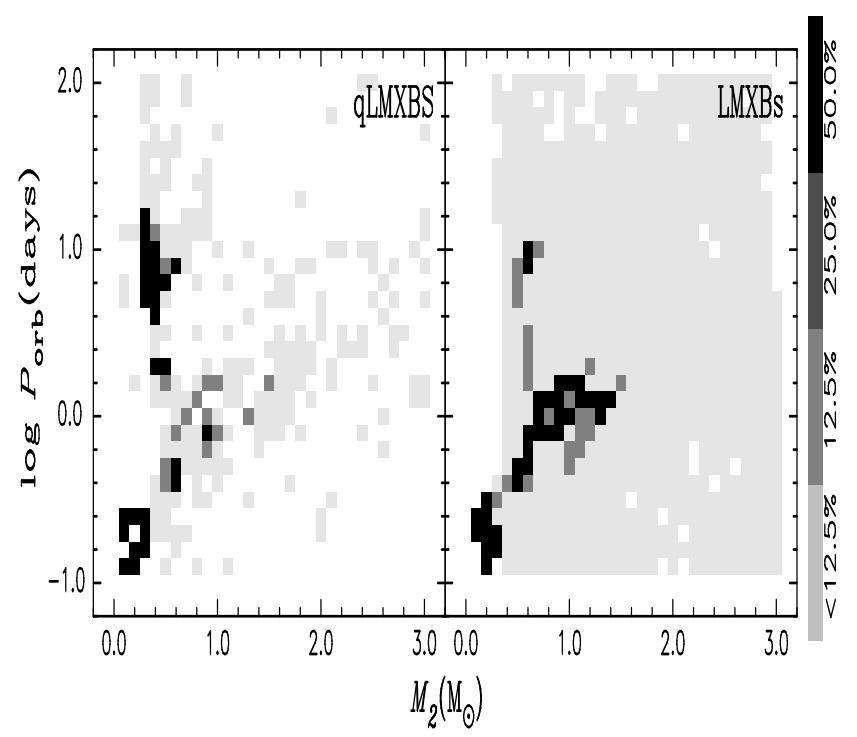

Fig. 5.- Similar to Figure 2, but for the distributions of the orbital periods $P_{\text {orb }}$ of qLMXBs and LMXBs vs. masses of their secondary stars, respectively.

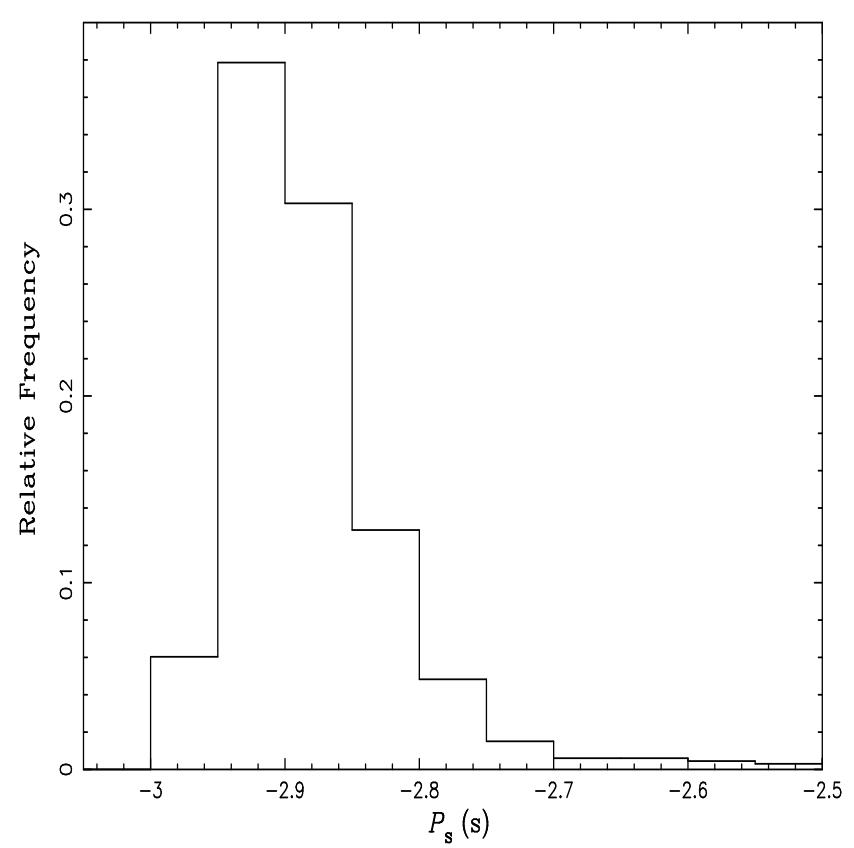

Fig. 6. - Number distribution of the spin periods of NSs at the beginning of the conversion of NS matter to SS matter. 
Demorest, P. B., Pennucci, T., Ransom, S. M., Roberts, M. S. E., \& Hessels, J. W. T. 2010, Nature, 467, 1081

Ergma, E., Sarna, M. J., \& Antipova, J. 1998, MNRAS, 300, 352

Ghosh, P., \& Lamb, F. K. 1978, ApJL, 223, L83

Haensel, P., Zdunik, J. L., \& Schaefer, R. 1986, A\&A, 160, 121

Han, Z. 1998, MNRAS, 296, 1019

Horvath, J. E., \& Benvenuto, O. G. 1988, Physics Letters B, 213, 516

Hurley, J. R., Pols, O. R., \& Tout, C. A. 2000, MNRAS, 315, 543

Hurley, J. R., Tout, C. A., \& Pols, O. R. 2002, MNRAS, 329, 897

Illarionov, A. F., \& Sunyaev, R. A. 1975, A\&A, 39, 185

Ivanova, N., Heinke, C. O., Rasio, F. A., Belczynski, K., \& Fregeau, J. M. 2008, MNRAS, 386, 553

Jonker, P. G., Steeghs, D., Nelemans, G., \& van der Klis, M. 2005, MNRAS, 356, 621

Kallman, T. R., Angelini, L., Boroson, B., \& Cottam, J. 2003, ApJ, 583, 861

Katz, J. I. 1975, Nat, 253, 698

Kiel, P. D., \& Hurley, J. R. 2006, MNRAS, 369, 1152

Kiel, P. D., Hurley, J. R., Bailes, M., \& Murray, J. R. 2008, MNRAS, 388, 393

Kluzniak, W. 1994, A\&A, 286, L17

Lai, X. Y., \& Xu, R. X. 2009, MNRAS, 398, L31

Lattimer, J. M., \& Prakash, M. 2007, Phys. Rep., 442, 109

Li, X.-D., Bombaci, I., Dey, M., Dey, J., \& van den Heuvel, E. P. J. 1999, Phys. Rev. Lett., 83, 3776

Lin, J., Rappaport, S., Podsiadlowski, P., Nelson, L., Paxton, B., \& Todorov, P. 2011, ApJ, 732, 70

Lipunov, V. M., Börner, G., \& Wadhwa, R. S. 1992, Astrophysics of Neutron Stars, ed. Lipunov, V. M., Börner, G., \& Wadhwa, R. S.

Lovelace, R. V. E., Romanova, M. M., \& Bisnovatyi-Kogan, G. S. 1995, MNRAS, 275, 244

—. 1999, ApJ, 514, 368 
Lü, G., Yungelson, L., \& Han, Z. 2006, MNRAS, 372, 1389

Lü, G., Zhu, C., Han, Z., \& Wang, Z. 2008, ApJ, 683, 990

Lü, G., Zhu, C., Wang, Z., \& Wang, N. 2009, MNRAS, 396, 1086

—. 2012, MNRAS, 124, 195

Madsen, J. 1988, Phys. Rev. Lett., 61, 2909

Miyaji, S., Nomoto, K., Yokoi, K., \& Sugimoto, D. 1980, PASJ, 32, 303

Nelson, L. A., \& Rappaport, S. 2003, ApJ, 598, 431

Olinto, A. V. 1987, Phys. Lett. B, 192, 71

Ouyed, R., Dey, J., \& Dey, M. 2002, A\&A, 390, L39

Ouyed, R., Staff, J., \& Jaikumar, P. 2011, ApJ, 729, 60

Ouyed, R., \& Staff, J. E. 2011, ArXiv:1111.3053

Pfahl, E., Rappaport, S., \& Podsiadlowski, P. 2002, ApJ, 573, 283

-. 2003, ApJ, 597, 1036

Podsiadlowski, P., Joss, P. C., \& Hsu, J. J. L. 1992, ApJ, 391, 246

Podsiadlowski, P., Rappaport, S., \& Pfahl, E. D. 2002, ApJ, 565, 1107

Pringle, J. E., \& Rees, M. J. 1972, A\&A, 21, 1

Serot, B. D., \& Uechi, H. 1987, Annals of Physics, 179, 272

Shakura, N., Postnov, K., Kochetkova, A., \& Hjalmarsdotter, L. 2012, MNRAS, 420, 216

Staff, J. E., Ouyed, R., \& Jaikumar, P. 2006, ApJ, 645, L145

Steeghs, D., \& Jonker, P. G. 2007, ApJ, 669, L85

van der Meer, A., Kaper, L., van Kerkwijk, M. H., Heemskerk, M. H. M., \& van den Heuvel, E. P. J. 2007, A\&A, 473, 523

Weber, F. 2005, Progress in Particle and Nuclear Physics, 54, 193

Wiringa, R. B., Fiks, V., \& Fabrocini, A. 1988, Phys. Rev. C, 38, 1010

Witten, E. 1984, Phy. Rew. D, 30, 272

Xu, R. X., Zhang, B., \& Qiao, G. J. 2001, Astroparticle Physics, 15, 101 
Yungelson, L. R., Tutukov, A. V., \& Livio, M. 1993, ApJ, 418, 794

Zhu, C., Lü, G., Wang, Z., \& Wang, N. 2012, PASP, 124, 195

This preprint was prepared with the AAS $\mathrm{LAT}_{\mathrm{E}} \mathrm{X}$ macros v5.2. 


\title{
Low-mass X-ray Binaries with Strange Quark Stars
}

\author{
Chunhua Zhu ${ }^{1,2,3}$, Guoliang "̈̈ $^{1,2}$, Zhaojun Wang ${ }^{1,2}$, Jinzhong Liu ${ }^{3}$ \\ ${ }^{\dagger}$ chunhuazhu@sina.cn
}

\begin{abstract}
Strange quark stars (SSs) may originate from accreting neutron stars (NSs) in lowmass X-ray binaries (LMXBs). Assuming that conversion of NS matter to SSs occurs when the core density of accreting NS reaches to the density of quark deconfinement, $\sim 5 \rho_{0}$, where $\rho_{0} \sim 2.7 \times 10^{14} \mathrm{~g} \mathrm{~cm}^{-3}$ is nuclear saturation density, we investigate LMXBs with SSs (qLMXBs). In our simulations, about 1\%o- $10 \%$ of LMXBs can produce SSs, which greatly depends on the masses of nascent NSs and the fraction of transferred matter accreted by the NSs. If the conversion does not affect binaries systems, LMXBs evolve into qLMXBs. We find that some observational properties (spin periods, X-ray luminosities and orbital periods) of qLMXBs are similar with those of LMXBs, and it is difficult to differ them. If the conversion disturbs the binaries systems, LMXBs can produce isolated SSs. These isolated SSs could be submillisecond pulsars, and their birthrate in the Galaxy is $\sim 5-70$ per Myr.

Subject headings: $\mathrm{b}$

inaries: close - stars: neutron-dense matter
\end{abstract}

\section{Introduction}

There are at least three different kinds of compact stars in the Universe: white dwarfs (WDs), neutron stars (NSs), and black holes. ? suggested a possible existence of compact objects consisting of strange quark matter. Due to strange quark matter being absolutely stable, ? and ? pointed out that NSs almost would be made of strange matter and not neutrons. However, ? considered that glitching radio-pulsars are NSs and not strange quark stars (SSs). ? suggested that SSs can not be formed directly in supernovaet, or less they would eventually contaminate the entire Galaxy. ?

\footnotetext{
${ }^{1}$ School of Physical Science and Technology, Xinjiang University, Urumqi, 830046, China.

${ }^{2}$ Xinjiang University-National Astronomical Observatories Joint Center for Astrophysics, Urumqi, 830046, China

${ }^{3}$ National Astronomical Observatories / Xinjiang Observatory, the Chinese Academy of Sciences, Urumqi, 830011, China

${ }^{1} \boldsymbol{?}$ and $?$ suggested that SSs can be formed directly during or shortly after some supernovae explosion when the central density of the proto-NSs is high enough.
} 
suggested that SSs could exist as millisecond pulsars. Due to the fast rotation and thermonuclear bursts, ? suggested that the SAX J1808.4-3658 is a good SS candidate. These SSs can be formed in low-mass X-ray binaries (LMXBs) via an accretion-triggered phase transition of NS matter to SS matter (?).

The phase transition requires the formation of a strange matter seed in the NS. The strange matter is produced through the neutron matter at a critical density. ? pointed out that the central density of a $1.4 M_{\odot}$ NS with a rather stiff equation of state is sufficiently lower than the critical density. Based on the modern equations of state in ?, ? estimated that the NSs with a $1.4 M_{\odot}$ must accrete matter of $\sim 0.5 M_{\odot}$ in order that their central densities reach the deconfinement density. Once the above condition is satisfied, the phase transition occurs.

? proposed that the process of the strange matter swallowing the neutron matter is a slow mode. However, ? showed that it is hydrodynamically unstable. ? proposed that the conversion of neutron matter should proceed in a detonation mode and could be accompanied by a gamma-ray burst. ? suggested that there is a quark-nova when the core of a neutron star (having experienced a transition to an up and down quark phase) shrinks into the equilibrated quark object after reaching strange quark matter saturation density (where a composition of up, down and strange quarks is the favored state of matter). In their model, the energy released as radiation in a quark-nova is up to $10^{53}$ ergs. ? proposed that the quark novae in LMXBs may be the engines of short gamma-ray bursts.

Based on the above descriptions, it is possible that SSs originate from the hydrodynamically unstable conversion or the slow conversion in LMXBs. Using standard equation of states of neutronrich matter, ? considered that the density of quark deconfinement is $\sim 5 \rho_{0}$, where $\rho_{0} \sim 2.7 \times 10^{14} \mathrm{~g}$ $\mathrm{cm}^{-3}$ is nuclear saturation density. According to the equation of states in ?, the gravitational mass of a NS is $\sim 1.8 M_{\odot}$ in order to reach $5 \rho_{0}$. Therefore, it is very important for our understanding of SSs' formation to study the mass evolution of NSs in LMXBs.

In this work, by simulating the interaction of a magnetized NS with its environment and utilizing a population synthesis code, we focus on the mass change of NSs in LMXBs and the possibility from NSs converting SSs in LMXBs, and investigate the properties of LMXBs with SSs (qLMXBs). In Section 2, we present our assumptions and describe some details of the modelling algorithm. In Section 3, we discuss the main results and the effects of different parameters. In Section 4, the main conclusions are given.

\section{Model}

For the simulation of binary evolution, we use rapid binary star evolution code BSE (?) with updates by ?. In interacting binaries, NSs can be formed via three channels(e.g., ??): (i) Corecollapse supernovae (CCSN) for a star; (ii)Evolution induced collapse (EIC) of a helium star with

a mass between 1.4 and $2.5 M_{\odot}$ in which the collapse is triggered by electron capture on ${ }^{20} \mathrm{Ne}$ and 
${ }^{24} \mathrm{Mg}$ (?); (iii) Accretion-induced collapses (AIC) for an accreting ONeMg WD whose mass reaches the Chandrasekhar limit. Response of accreting ONeMg WD is treated in the same way as the evolution of CO WD (see details in ?).

\subsection{Mass of Nascent Neutron Star}

Possibly the masses are one of the most important properties of NSs. However, the mass distribution of nascent NSs are not yet well known. In BSE code, the gravitational mass of a nascent NS via CCSN depends on the mass of the CO-core at the time of supernova (?). Figure 1 shows the masses of nascent NSs forming from different initial masses. Some authors assumed that the initial masses of NSs $\left(M_{\mathrm{NS}}^{\mathrm{i}}\right)$ are $1.4 M_{\odot}$ in their works (???, e.g.,). ? showed that the masses of some NSs are lower than $1.4 M_{\odot}$. Recently, ? found that the masses of NSs in SMC X-1 and

Cen X-3 are $1.06_{-0.10}^{+0.11} M_{\odot}$ and $1.34_{-0.14}^{+0.16} M_{\odot}$, respectively. However, It is well known that most of the accurately measured masses of NSs are near $1.4 M_{\odot}$.

In our work, we use the initial masses of NSs via CCSN in ? and $M_{\mathrm{NS}}^{\mathrm{i}}=1.4 M_{\odot}$ in different simulations, respectively. For NSs via AIC, following ?, we take $M_{\mathrm{NS}}^{\mathrm{i}}=1.3 M_{\odot}$. Similarly, for NSs via EIC, we also take $M_{\mathrm{NS}}^{\mathrm{i}}=1.3 M_{\odot}$.

In addition, nascent NS receives additional velocity ("kick") due to some still unclear process that disrupts spherical symmetry during the collapse or later Dichotomous nature of kicks which was suggested quite early by ?. Observationally, the kick is not well constrained due to numerous selection effects. Currently, high kicks $\left(\sim 100 \mathrm{~km} \mathrm{~s}^{-1}\right)$ are associated with NS originating from CCSN, while low kicks $\left(\sim 10 \mathrm{~km} \mathrm{~s}^{-1}\right)$ with NS born in EIC and AIC (?). We apply to core-collapse NS Maxwellian distribution of kick velocity $v_{\mathrm{k}}$

$$
P\left(v_{\mathrm{k}}\right)=\sqrt{\frac{2}{\pi}} \frac{v_{\mathrm{k}}^{2}}{\sigma_{\mathrm{k}}^{3}} e^{-v_{\mathrm{k}}^{2} / 2 \sigma_{\mathrm{k}}^{2}}
$$

Variation of $\sigma_{\mathrm{k}}$ between 50 and $200 \mathrm{~km} \mathrm{~s}^{-1}$, introduces an uncertainty $\lesssim 3$ in the birthrate of lowand intermediate-mass X-ray binaries (?). ? discussed the effects of parameter $\sigma_{\mathrm{k}}$ on LMXBs' populations. Since in this paper we focus on the physical parameters that mostly affect the masses of NS, we do not discuss the effects of $\sigma_{\mathrm{k}}$ on SSs' population. Following ?, we take $\sigma_{\mathrm{k}}=190 \mathrm{~km}$ $\mathrm{s}^{-1}$ in CCSN, and $\sigma_{\mathrm{k}}=20 \mathrm{~km} \mathrm{~s}^{-1}$ in EIC and AIC.

\subsection{Mass of Accreting Neutron Star}

In LMXBs, NSs accrete the matter from their companions via Roche lobe flows or stellar winds. The interaction of a rotating magnetized NS (single or in a binary system) with surrounding matter has been studies by many authors (?????, e. g.,). 
Using a convenient way of describing NS evolution elaborated by ? and a recent model for quasi-spherical accretion including subsonic settling proposed by ?, ? gave a detailed simulations for spin period evolution and matter accretion of NSs in binaries. In this work, we adopt their model. ? assumed that all matter transferred is accreted by the NS in an accretor stage. We introduce a parameter $\beta$ which is the fraction of transferred matter accreted by the NS, and the rest of the transferred mass is lost from binary system. The lost matter takes away the specific angular momentum of the prospective donor. The value of $\beta$ has been usually set to 0.5 (???). In our work, we set $\beta=0.1,0.5$ and 1.0 in different simulations.

\subsection{LMXBs with Nascent SSs}

As noted in $£$, when the gravitational mass of a NS reaches to $\sim 1.8 M_{\odot}$, the NS can turn into a SS via the hydrodynamically unstable conversion or the slow conversion. In the former mode, the binary system may been disrupted (?), and it becomes two isolated star. One of them is an isolated SS. However, it is difficult to know the effects of the hydrodynamically unstable conversion on binary systems. Therefore, in our work, we consider two extreme cases: (i) In order to simulate all potential properties of qLMXBs, we assumed all LMXBs are not affected and survive after the conversion, that is, LMXBs become qLMXBs when the masses of accreting NSs are larger than $1.8 M_{\odot}$; (ii) We assumed that all LMXBs are disrupted after the conversion, that is, there is no qLMXBs. However, we can discuss the origin of isolated submillisecond pulsars.

In our simulations, if a nascent NS has larger mass than $1.8 M_{\odot}$ it is a SS. Therefore, in the paper, qLMXBs include some LMXBs in which the nascent NSs have larger mass than $1.8 M_{\odot}$. ? suggested that SSs could have high maximum masses (See Figure 2 in ?) if they are composed of the Lennard-Jones matter. In our work, we assume that the maximum mass of SS is $3.0 M_{\odot}$.

\section{Results}

We use Monte Carlo method to simulate the initial binaries. For initial mass function, massratios, and separations of components in binary systems, we adopt the distributions used by us in ??. We assume that all binaries have initially circular orbits. After a supernova, new parameters of the orbit are derived using standard formulae, (e. g., ?). It is well known that theoretical models of the population of LMXBs depend on badly known input parameters, such as kick velocity and common envelope treatment (e. g., ??). However, in this pioneering study of qLMXBs we focus on the effects which are important for the masses of NSs: the efficiency accreted of transferred matter, $\beta\left(\beta=0.1,0.5\right.$ and 1.0 ), and the mass of nascent NS via CCSN (See Figure 1 ). We use $1 \times 10^{8}$ binary systems in our Monte-Carlo simulations. 


\subsection{Mass Increases of Accreting NSs}

Figure 2 gives the mass increases of accreting NSs in LMXBs. According the assumptions of SSs formatting from NSs with masses higher than $1.8 M_{\odot}, 1 \%(\beta=0.1)-10 \%(\beta=1.0)$ of LMXBs are qLMXBs in our simulations. This proportion greatly depends on input parameters $\beta$ and the initial masses of nascent NSs. In the cases of $\beta=1.0$ and $\beta=0.5$, most of SSs in qLMXBs come from low-mass NSs with $1.4 M_{\odot}$, that is, they have accreted $\sim 0.4 M_{\odot}$ matter. In the case of $\beta=0.1$, most of SSs in qLMXBs originate from CCSN.

Mass increases of accreting NSs in LMXBs depend on not only input parameter $\beta$ in this work, but also orbital periods and NSs' companions. ? showed that most of NSs in LMXBs with WD donors have low mass-accretion rates $\left(\sim 10^{-12} \dot{M}_{\odot} \mathrm{yr}^{-1}\right)$ and most of LMXBs with WD donors are transient. Therefore, the masses of accreting NSs in LMXBs with WD donors hardly reach to 1.8 $M_{\odot}$. Less than $10 \%$ (in case of $\beta=0.1$ ) $-1 \%$ o (in case of $\beta=0.5, M_{\mathrm{NS}}^{\mathrm{i}}=1.4 M_{\odot}$ ) of qLMXBs have WD donors in our simulations. Most of qLMXBs have MS donors. From now on, we just discuss LMXBs and qLMXBs with MS donors.

\subsection{Properties of $q \mathrm{LMXBs}$}

According to our assumption that the conversion of NS matter to SS matter does not affect binary systems, we can simulate some observational properties of LMXBs and qLMXBs, and wish to find some differences between them. Then, taking the case of $\beta=0.5$ for example, we discuss the some properties of qLMXBs.

X-ray luminosities (mass-accretion rates), spin periods and orbital periods are important parameters of LMXBs. Figure 3 shows the accretion rates by NSs or SSs in LMXBs and qLMXBs, and the X-ray luminosities which are approximated as

$$
L_{\mathrm{x}}=\eta \dot{M}_{\mathrm{NS}} \mathrm{c}^{2} \simeq 5.7 \times 10^{35} \mathrm{erg} \mathrm{s}^{-1}\left(\frac{\eta}{0.1}\right) \times\left(\frac{\dot{M}_{\mathrm{NS}}}{10^{-10} M_{\odot} \mathrm{yr}^{-1}}\right),
$$

where $\eta \simeq 0.1$ is the efficiency of converting accreted mass into X-ray photons. We can find that there is not significant difference between accretion rates by NSs or SSs in LMXBs and qLMXBs.

The spin periods of the rotating magnetized NSs mainly depend on their mass-accretion rates (?). If accreting SSs are similar to NSs, we can simulate the spin periods of accreting SSs. Figure 4 gives the distribution of the spin periods of NSs in LMXBs or SSs in qLMXBs. In general, spin periods of SSs in qLMXBs are shorter than those of NSs in LMXBs.

Figure 5 shows the the distributions of the orbital periods $P_{\text {orb }}$ of qLMXBs and LMXBs vs. masses of their secondary stars. Similarly, there is not significant difference between qLMXBs and LMXBs.

As the above descriptions show, it is very difficult in our model to differ from LMXBs and 


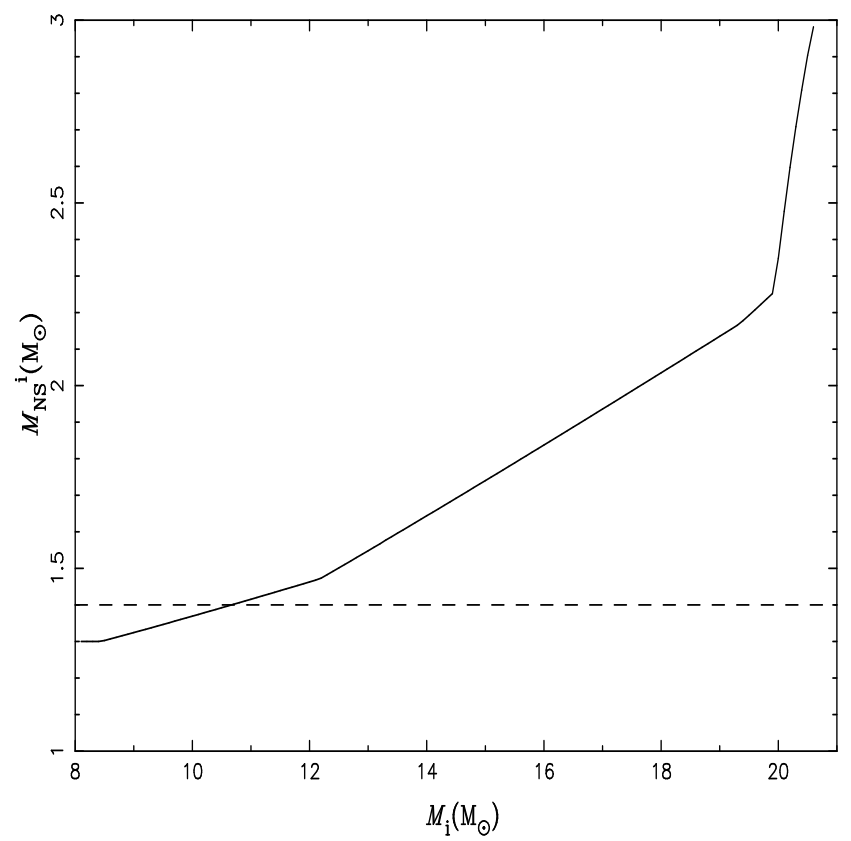

Fig. 1.- Masses of nascent NSs via CCSN vs. stellar initial masses. The solid line comes from ?, and the dashed line means that masses of nascent NSs are equal to $1.4 M_{\odot}$.

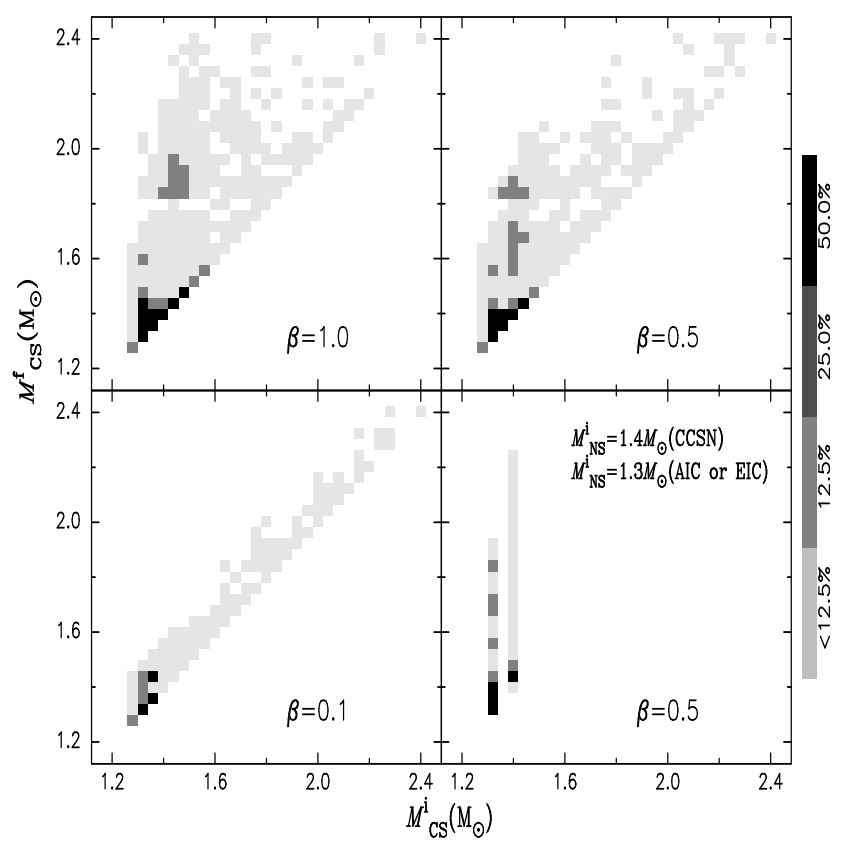

Fig. 2.- Distributions of the initial masses of compact stars (NSs or SSs) vs. their final masses in LMXBs for different input parameters. Gradations of gray-scale correspond to the number density of systems $>1 / 2,1 / 2-1 / 4,1 / 4-1 / 8,1 / 8-0$ of the maximum of $\frac{\partial^{2} N}{\partial M_{\mathrm{CS}}^{\mathrm{i}} \partial M_{\mathrm{CS}}^{\mathrm{f}}}$ and blank regions do not contain any stars. 


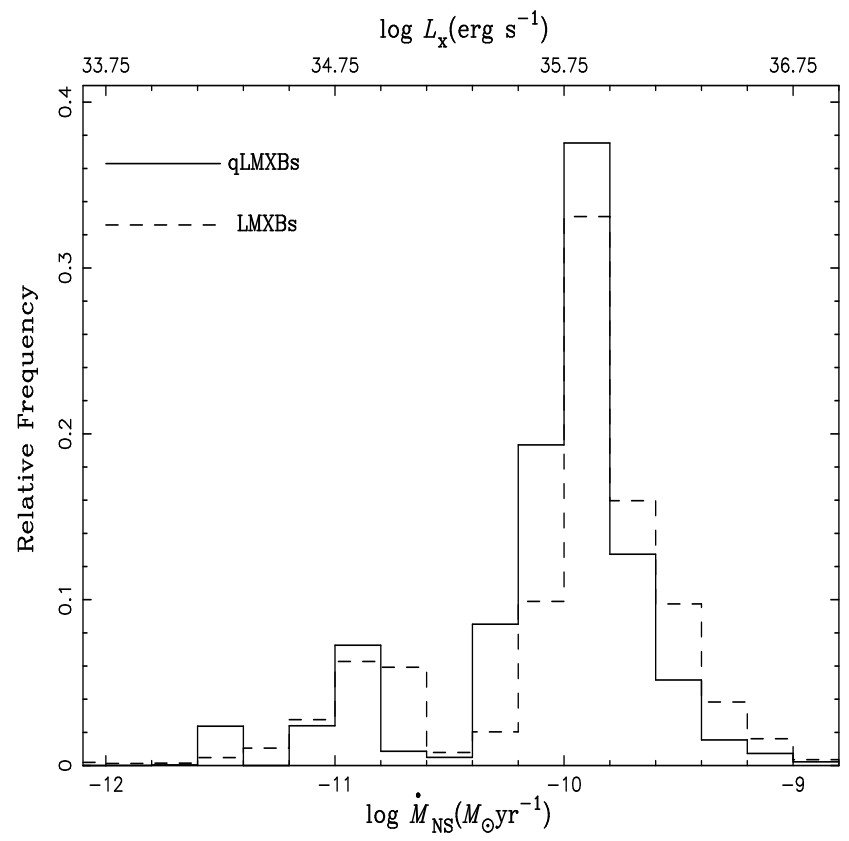

Fig. 3.- Distributions of accretion rates (X-ray luminosities) by NSs or SSs in LMXBs and qLMXBs, respectively.

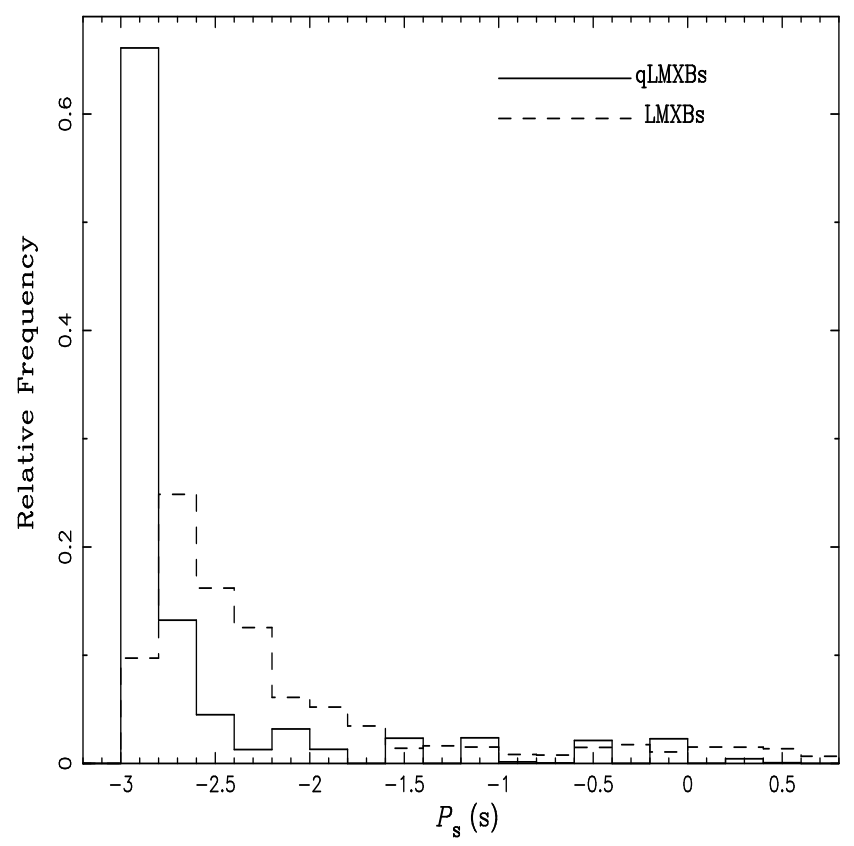

Fig. 4. - Number distributions of the spin periods of NSs or SSs in LMXBs and qLMXBs, respectively. The numbers are normalized to 1 . 
qLMXBs. The most effective way is to measure the masses of compact objects in LMXBs if the assumption that NSs with masses larger than $1.8 M_{\odot}$ are SSs is right.

? suggested that compact object in 2S 0921-630 (It is a LMXB) has a mass between $\sim 1.9-2.9$ $M_{\odot}$. According to our assumption, the compact object is a SS. However, ? considered that ? overestimated the rotational broadening and the mass of compact object in $2 \mathrm{~S} 0921-630$ is $\sim 1.44$ $M_{\odot}$. The orbital period of $2 \mathrm{~S} 0921-630$ is $9.006 \pm 0.007$ days, and its X-ray luminosity is $\sim 10^{36} \mathrm{erg}$

$\mathrm{s}^{-1}($ ?). Results of simulating LMXBs and qLMXBs in our work both cover these observations. Therefore, we can not conclude whether compact object in 2S 0921-630 is NSs or SSs.

? gave that PSR 1614-2230 has a mass of $1.97 M_{\odot}$. And, it is a millisecond radio pulsar (Pulsar spin period is $3.15 \mathrm{~ms}$ ) in an 8.7 day orbit, and its companion has a mass of $0.5 M_{o}$ dot. Although PSR 1614-2230 is not X-ray binary, it may come from X-ray binary. ? suggested that PSR 1614-2230 descended from an LMXB very much like Cyg X-2 ( $P_{\text {orb }}=9.8$ days, $M_{\mathrm{NS}}=1.7 M_{\odot}$ and $M_{2}=0.6 M_{\odot}$, see ?). Figure 5 shows the distributions of the orbital periods $P_{\text {orb }}$ of qLMXBs and LMXBs vs. masses of their secondary stars. Our results cover the positions of orbital periods and companion masses of Cyg X-2 and radio millisecond pulsar binary PSR 1614-2230. Our work support that PSR 1614-2230 originate from a LMXB. Now, PSR 1614-2230 may be a SS.

\subsection{Submillisecond Pulsars}

? suggested that an isolated submillisecond pulsar spinning at $\sim 0.5 \mathrm{~ms}$ could strongly hint the existence of SSs. If the conversion of NS matter to SS matter is hydrodynamically unstable and LMXBs are disrupted, the nascent SSs are isolated. Assuming that one binary with $M_{1} \geq 0.8 M_{\odot}$ is formed per year in the Galaxy (???), we can estimate that the occurrence rate of hydrodynamically unstable conversion is about $5-70$ per Myr.

As Figure 6 shows, the majority of NSs at the beginning of the conversion have spin periods longer than $1 \mathrm{~ms}$. If the angular momentum is conserved and no unknown physical mechanic spins up NSs and nascent SSs during the conversion, the spin periods of nascent SSs depend on the change of moment of inertia. ? considered that a typical ejected mass of hydrodynamically unstable conversion is $\sim 10^{-3} M_{\odot}$. Therefore, the change of moment of inertia is determined by the difference of radius between NS and SS. ? estimated that NS could shrink by as much as $30 \%$. Then, many nascent SSs have spin periods of $\sim 0.5 \mathrm{~ms}$ in our simulations. However, this result greatly depends on the equation of state of NSs and SSs which are poorly known. If NS only shrinks by as much as $10 \%$ (Private discussion with $\mathrm{Xu}$ ), it is difficult for the nascent SSs to spin up to 0.5 ms. 


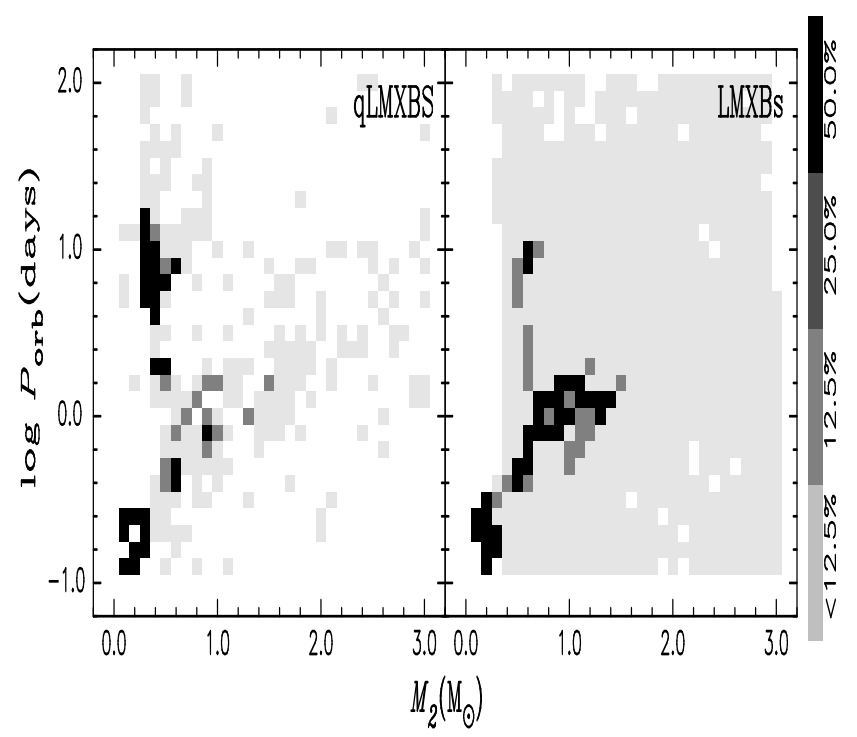

Fig. 5.- Similar to Figure 2, but for the distributions of the orbital periods $P_{\text {orb }}$ of qLMXBs and LMXBs vs. masses of their secondary stars, respectively.

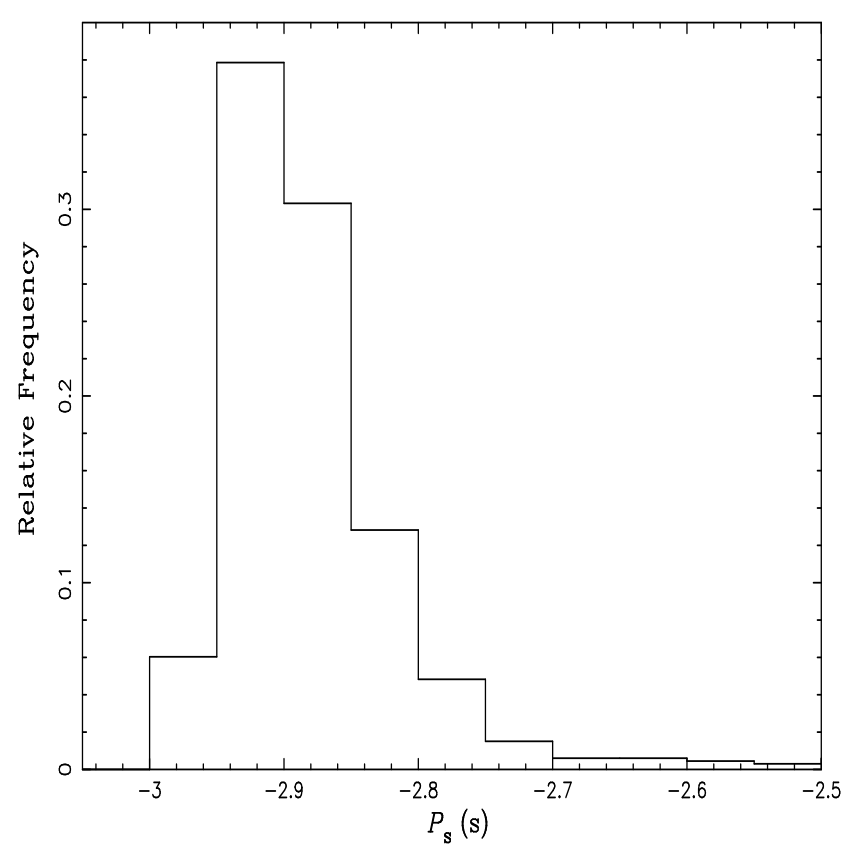

Fig. 6. - Number distribution of the spin periods of NSs at the beginning of the conversion of NS matter to SS matter. 


\section{Conclusions}

Employing the population synthesis approach to the evolution of binaries and using the interacting model of a rotating magnetized NS with surrounding matter, we investigate the mass change of NSs in LMXBs and the possibility from NSs converting SSs in LMXBs. Our results shows that about $1 \% 0-10 \%$ of LMXBs can produce SSs. These SSs may exist in qLMXBs or be isolated, which depends on physical model of the conversion of NS matter to SS mater.

Our toy model can not conclude whether there are SSs in the Galaxy and can not give what

properties qLMXBs have. In further work, we need detailed physical model (equation of state about NS and SS and the conversion of NS matter to SS mater) to improve our work.

\section{Acknowledgments}

This work was supported by the National Natural Science Foundation of China under Nos. 11063002 and 11163005, Natural Science Foundation of Xinjiang under Nos.2009211B01 and 2010211B05, Foundation of Huoyingdong under No. 121107, Foundation of Ministry of Education under No. 211198, and Doctor Foundation of Xinjiang University (BS100106). 